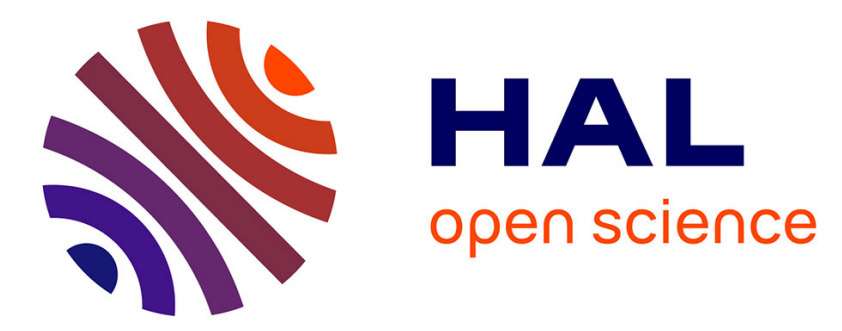

\title{
Introduction. L'arc latin, histoire et problématiques d'un concept
}

\author{
Sylvie Daviet
}

\section{To cite this version:}

Sylvie Daviet. Introduction. L'arc latin, histoire et problématiques d'un concept. Méditerranée : revue géographique des pays méditerranéens, 1994, 79, pp.3 - 6. 10.3406/medit.1994.1844 . hal-03203757

\section{HAL Id: hal-03203757 \\ https://hal.science/hal-03203757}

Submitted on 21 Apr 2021

HAL is a multi-disciplinary open access archive for the deposit and dissemination of scientific research documents, whether they are published or not. The documents may come from teaching and research institutions in France or abroad, or from public or private research centers.
L'archive ouverte pluridisciplinaire HAL, est destinée au dépôt et à la diffusion de documents scientifiques de niveau recherche, publiés ou non, émanant des établissements d'enseignement et de recherche français ou étrangers, des laboratoires publics ou privés. 


\section{Introduction. L'arc latin, histoire et problématiques d'un concept}

Mme Sylvie Daviet

\section{Citer ce document / Cite this document :}

Daviet Sylvie. Introduction. L'arc latin, histoire et problématiques d'un concept. In: Méditerranée, tome 79, 1-2-1994. L 'arc méditerranéen en questions. pp. 3-6;

doi : 10.3406/medit.1994.1844

http://www.persee.fr/doc/medit_0025-8296_1994_num_79_1_1844

Document généré le 13/06/2016 
INTRODUCTION

\section{L'arc latin, histoire et problématiques d'un concept}

\section{Sylvie DAVIET*}

Le géographe, comme l'économiste ou le sociologue..., recèle en lui trois personnages : le scientifique soucieux d'établir la vérité des faits à l'aide d'indicateurs irréfutables, l'homme de son temps qui voit le monde avec toute l'épaisseur de sa culture sociale et régionale, le citoyen porteur de valeurs et d'un projet de société. Certains débats, parce qu'ils touchent un point sensible de notre identité, suscitent plus que d'autres la confusion des genres. Le débat sur l'arc latin est de ceux-là.

La genèse du concept a naturellement des points communs avec celle de l'arc atlantique dont il est le frère (Norois, 1993). A l'origine se trouve la Conférence des Régions Périphériques Maritimes (CRPM) née en 1973 d'une initiative d'Olivier Guichard (1), et qui rassemble 65 régions de la Communauté allant du Danemark à la Grèce. Son objectif est de «faire contre-poids aux grandes concentrations humaines et économiques du centre de l'Europe». Puis en 1987, le «rapport Guichard» préconise le renforcement, en dehors de la région parisienne, de deux ou trois pôles de croissance capables de soutenir la compétition internationale. Le second élément déterminant, en cette fin des années 1980, est donc la préparation du grand marché unique de 1993. La Chambre Régionale de Commerce et d'Industrie de Provence-Alpes-Côte d'Azur inscrit à l'ordre du jour de son assemblée plénière l'analyse de ce rapport, et produit un document sans complaisance (JUAN, 1987) sur les faiblesses de la métropole marseillaise face au triangle Barcelone-Lyon-Milan. Le troisième élément qui vient stigmatiser les représentations en cours est sans conteste la série de cartes élaborées par le groupe GIP-RECLUS (BRUNET, 1989), mettant en évidence, après E. JUILLARD (1976), une dorsale qui structure l'espace européen de Londres à Milan via l'axe rhénan. La chute du mur de Berlin ne fera qu'accentuer à l'Ouest les craintes d'une marginalisation. En 1989, les associations constitutives de l'arc atlantique et latin voient le jour, mais connaîtront, malgré des analogies certaines, des destins quelque peu divergents; l'une est le fait d'hommes politiques influents, l'autre est portée par des socio-professionnels.

La thèse du contre-poids a, en définitive, présidé à la naissance de l'arc. C'est là un thème récurrent en géographie, notamment en géographie des frontières où l'équilibre des forces relève d'une préoccupation quasi-militaire. L'idée d'un rééquilibrage puise par ailleurs sa légitimité dans une conception implicitement cartésienne de l'harmonie des formes et des espaces, lesquels devraient s'ordonner autour d'un axe imaginaire de symétrie. La configuration proposée par R. BRUNET, qui substitue la notion de faisceau à celle d'arc (Fig. 1), illustre parfaitement cette démarche joignant à l'esthétique des lignes -le territoire est dépouillé du trait de côte- un sens mesuré de l'équité : «l'Europe entière, et pas seulement le Sud-Ouest, gagnera à être moins inégalitaire, plus polycentrique» (BRUNET, 1993). Mais elle va plus loin : en exprimant une inversion radicale des valeurs, elle fait du Sud-Ouest, le nouveau centre de l'art de vivre face à l'arc périphérique d'une dorsale grise et industrieuse : «Les deux arcs présentent ensemble la perspective de changer de siècle : d'offrir le passage de l'Europe du charbon, des fumées et des brumes à une Europe des villes ouvertes sur des environnements de qualité». Au-delà de sa dimension anthropologique, le concept d'arc (atlantique ou latin) est donc éminemment stratégique, et les géographes auraient sans doute tort d'abandonner ce terrain à d'autres, mais le projet n'a de chance de se réaliser que s'il s'inscrit dans une logique des faits.

Si nous analysons maintenant les diagnostics proposés de l'espace français voire européen, nous nous trouvons en présence de lectures contradictoires des dynamiques territoriales. $D$ 'un côté, la nouvelle orthodoxie francilienne des années 1990 du «tout à la mégapole» (BENKO, LIPIETZ, 1992) se trouve justifiée par la capacité des grandes métropoles à concentrer et générer de nouveaux processus interactifs. Le point de vue marxiste rejoint le point de vue francilien dans la mesure où, s'appuyant sur les hiérarchies du système

* Maître de Conférences, Institut de Géographique, Université de Provence, Aix-en-Provence.

1 - Olivier Guichard, ancien ministre gaulliste, est actuellement président du Conseil régional de Pays de Loire. 


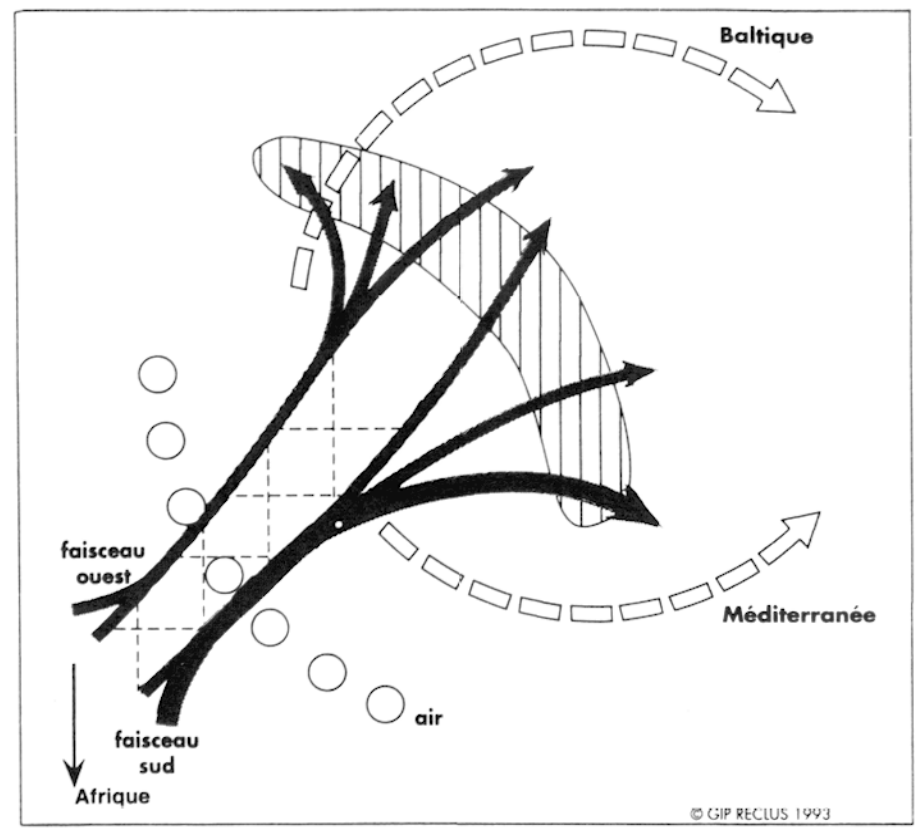

Source: R. BRUNET(1993),- L'arc atlantique, son reflet, ses mirages, Norois, $\mathrm{N}^{\circ} 157$, p. 19-34.

«Mieux et autrement que deux arcs, l'avenir est à deux grands faisceaux de communication unissant la Péninsule ibérique au reste de l'Europe. Leur équipement est un enjeu majeur et immédiat de la construction européenne. Leur laçage s'impose si l'on cherche à réquilibrer l'Europe au sudouest, et àrevitaliser les espaces intermédiaires. Il faudra y ajouter des politiques énergétiques de navigation maritime et aérienne vers les autres Europes et les continents voisins, afin de contourner la Mégapole au lieu de la renforcer».

Fig. 1 - LES GRANDS FAISCEAUX DE L'EUROPE DE L'OUEST.

productif (production/reproduction sociale), il ne voit émerger que la capitale, et enregistre un encéphalogramme plat à l'endroit des métropoles régionales, à l'exception de Lyon (Beckouche, DametTe, 1994). D'une façon générale, les analyses d'économistes convergent vers un constat de dépendance du Sud français. De l'autre côté, la mise en évidence de nouveaux espaces de croissance, d'un «modèle Sud», est le fruit d'un courant plus méridional, s'appuyant sur des indicateurs de croissance de la population et de l'emploi (BERGER, 1993; De Los Santos, 1993), des phénomènes de métropolisation de la façade du grand delta (FerRier, 1993), désormais repris par la DATAR (Ministère de l'Intérieur, 1993), une évolution des comportements favorable à l'innovation (SCARDIGL, 1992). Comment une dynamique démographique, territoriale, sociale, peut-elle s'affirmer sans générer de réelle dynamique économique ?.Il faut sans doute s'interroger sur les raisons d'un tel divorce entre les composantes d'un même système spatial. L'article de F. Blanc, C. CENTI, L. URDY : «Intégration spatiale et arc latin» (dans ce numéro) apporte à cet égard des éléments de réponse, en mettant l'accent sur les dysfonctionnements d'un système qui modèle les comportements et oriente les flux; en France le couple localisme/néocentralisme est ainsi montré du doigt. A l'échelle de l'arc, on assiste à la juxtaposition de modes de développements régionaux et nationaux présentant de multiples fractures (CEE, 1993) : le cas espagnol oppose la Catalogne, orientée vers le marché, et l'Andalousie qui s'apparente au Mezzogiorno par l'importance des investissements publics et des transferts sociaux. La façade italienne est éclatée entre un couloir ligure tourné vers le nord, le modèle toscan de la troisième Italie et la métropole romaine dépourvue de réel rayonnement économique.

On le voit, la controverse est de rigueur. Pourtant, la notion d'arc a acquis une certaine autonomie. Il est vrai que «nommer c'est déjà construire» (RUEL, 1991). En intégrant les représentations établies par J.F. DeVRet (1988) et R. BRUNEt (1989), la Communauté a procédé à un découpage de l'espace européen en zones faisant l'objet d'études prospectives. L'arc atlantique bénéficie désormais d'un véritable programme en sa faveur : Atlantis. L'arc latin est dans l'attente. Ses limites géographiques sont à géométrie variable : il s'étend, pour les uns, de Valence à Rome (nord du Sud), pour les autres, du détroit de Gibraltar à celui de Messine (Vorron, 1993), il forme, pour la CEE, un arc dissymétrique allant de l'Andalousie au Latium, excluant Naples et la botte italienne (Fig. 2).

A l'inverse de son concurrent, il n'a pas suscité de véritable «lobbying» chez les politiques. La Méditerranée n'est pas l'Atlantique, le jeu des acteurs y est plus complexe. Il ne peut ignorer, en dépit de ses insuffisances, son rôle d'interface avec les rives méridionales et orientales du bassin. Parmi les liens que l'arc latin entretient avec les pays méditerranéens, les liens migratoires dominent, les liens commerciaux et financiers sont plus lâches et tendent à s'amenuiser (CEFI, 1992). Il en est de même à l'échelle européenne, mais c'est l'Europe du Nord et non du Sud qui occupe une position privilégiée dans ses échanges avec les pays du Sud-Est méditerranéen (CEE, 1992). La problématique de l'interaction est ainsi entrée dans le champ des préccupations communautaires et des collectivités territoriales. Utilisant les opportunités de la «Politique méditerranénne rénovée» de la CEE, la Mission Internationale de la ville de Marseille, et le Conseil régional 
de Provence-Alpes-Côte d'Azur - à travers la Charte du bassin méditerranéen-, semblent renouer avec une longue tradition d'échanges et de coopération en proposant aux acteurs de l'autre rive un réseau d'experts et de services en matière d'ingénierie urbaine, de transfert de technologie ou d'environnement. Au moment où les conflits en Bosnie, en Algérie, laissent planer dans la région la menace d'un nouveau «rideau de fer», la libéralisation de l'Europe centrale, l'émergence de la Turquie, les espoirs de paix au Proche-Orient... peuvent activer des flux aux conséquences multiples. Les incertitudes qui pèsent sur l'avenir de l'espace méditerranéen n'autorisent pas l'absence de stratégie. En ce sens, le modèle proposé par R. BRUNEr (Fig. 1) intègre à juste titre cet éventail de perspectives.

Enfin, la question des fondements historiques et culturels qui sous-tendent la thèse de l'arc mérite des investigations nouvelles et spécifiques. Pour R. CATY et E. Richard, qui se prêtent ici à cet exercice, il existe bien au XIX ${ }^{e}$ siècle des éléments économiques et humains constitutifs de l'arc latin. Et nous pourrions suivre d'autres pistes en interrogeant notamment l'histoire des mouvements migratoires. Pourtant, on ne voit pas s'affirmer d'identité latine. Nos histoires nationales en ont sans doute segmenté les bases. La fragilité de l'identité latine résulte aussi de la force même du mythe globalisant de la Méditerranée. La fractionner en ensembles géopolitiques indépendants les uns des autres, c'est renoncer au rêve andalou d'osmose entre

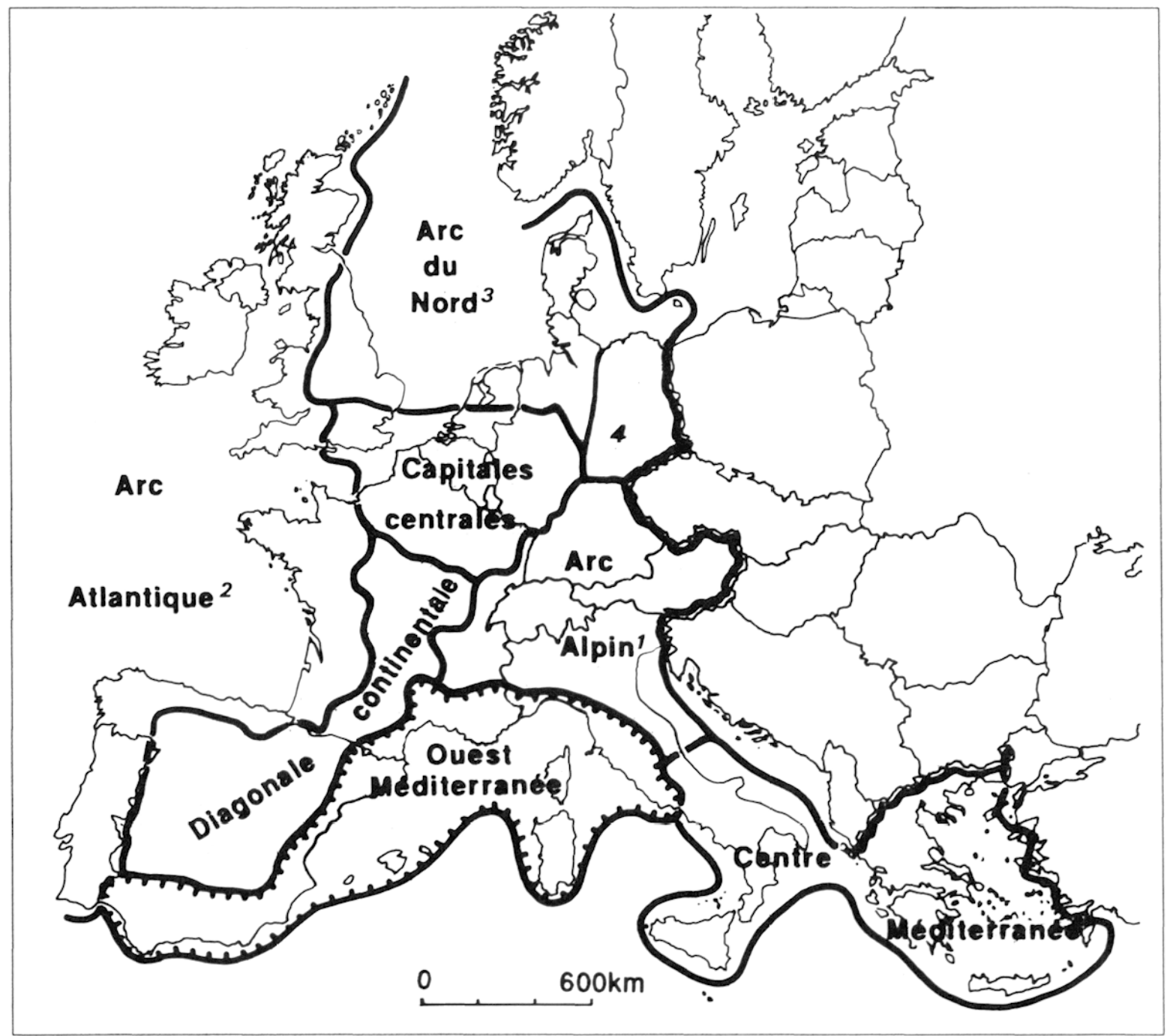

Fig. 2 - ZONES D'ÉTUDES TRANSRÉGIONALES DE LA CEE.

$1:$ avec les parties alpines de Provence-Alpes-Côte d'Azur; $2:$ avec des zones d'Andalousie occidentale; $3:$ avec l'Ecosse orientale et la lande de Mecklenburg-Vorpommern; 4 : Nouveaux Länder.

Source : Commission des Communautés Européennes, Direction générale des politiques régionales, (1991).- Europe 2000 les perspectives de développement du territoire communautaire, p. 23.

La configuration proposée ici se rapproche davantage du modèle établi par J.F. Drevet (1988), dans lequel «l'Europolygone des capitales» se substitue à la dorsale comme espace central. On note la multiplication de terme d' «arc»; curieusement celui d'arc latin n'y figure pas. Le littoral méditerranéen est scindé en deux : le Centre Méditerranée n'est pas sans rappeler la Grande Grèce, l'Ouest Méditerranée, amputée de la botte italienne et de la Sicile, forme un arc dissymétrique dont la cohérence pose problème. 
les religions, les cultures, les hommes. Entre la grande Méditerranée et les morceaux de Méditerranée catalane, provençale ou ligure, la «néo-latinité» peut apparaître comme le produit d'une culture européocentrique qui se définit davantage par rapport à la germanité qu'en référence au monde arabe (HeNRY, 1993).

De fait, l'arc latin souffre d'un défaut d'articulation entre sa dimension européenne et sa dimension méditerranéenne. Les deux visions émanent de sensibilités, de logiques différentes et se tournent le plus souvent le dos. L'étude prospective de X. Gizard (1993) présente, de ce point de vue, l'intérêt de souligner la communauté de destins qui lie, pour le meilleur et pour le pire, l'Europe du sud aux pays du Sud-Est méditerranéen. Rives nord de la Méditerranée, rives sud de l'Europe, l'arc latin est bien un arc de contradictions entre le Nord-le Sud, le passé-le présent, le rêve-la réalité. Son manque de lisibilité, son incapacité à se laisser enfermer dans les limites géographiques précises ne sont que les signes d'une réalité plastique, et ne sauraient disqualifier la pertinence du sujet. Ce dernier pose plus largement deux questions majeures à l'Europe d'aujourd'hui. La première relève de son organisation interne : quelle sera sa capacité à intégrer de nouvelles échelles de fonctionnalités pour construire de véritables ensembles régionaux transfrontaliers? La seconde, d'ordre géopolitique, relève du débat entre régionalisme et mondialisme (Hugon, 1993) : l'Union Européenne doit-elle persévérer dans une logique de mondialisation et d'affaiblissement de ses relations avec le monde méditerranéen tandis que se constituent autour des Etats-Unis (avec l'ALENA) et du Japon (avec l'ASEAN et les nouveaux dragons) des espaces régionaux dynamiques? En d'autres termes, l'arc latin peut-il êre le vecteur d'une «Méditerranée réinventée» (BALTA, 1992)?

\section{BIBLIOGRAPHIE}

Balta P. sous la dir. de, (1992).- La Méditerranée réinventée, réalités et espoirs de la coopération. La Découverte/ Fondation René Seydoux, $393 \mathrm{p}$.

Beckouche P., Damette F., (1994).- Economie, société et territoires, une grille d'analyse globale de l'emploi, in Economie \& Statistiques, à paraître.

Benko G., LipIETz A., (1992).- Les régions qui gagnent, PUF, Paris, 424 p.

Berger A., (1993).- Les nouveaux espaces de croissance dans l'Europe du Sud, in L'adhésion de l'Espagne et du Portugal à la CEE, Maison des Pays ibériques, p.61-75.

BRUNET R., (1989).- Les villes européennes, DATAR-RECLUS, 79 p.

Brunet R., (1993).- L'arc atlantique, son reflet, ses mirages, Norois, No 157, p.19-34.

CEE, (1992).- Impact sur le développement régional et l'aménagement de l'espace communautaire des pays du sud et de l'est méditerranéen, Rapport final provisoire, $216 \mathrm{p}$.

CEE, (1993).- Evolution prospective des régions de la Méditerranée Ouest, DG XVI, Rapport final, 216 p.

CEFI, 1992.- La Méditerranée économique, Economica, 576 p.

-De Los SANtos A., (1993).- Retour au Sud, un succès en déséquilibres, La Méditerranée inquiète, DATAR-AUBE, p. 123 130.

DREvet J.F., (1988).- Les régions françaises entre l'Europe et le déclin, Souffles, 253 p.

FerRier J.P., (1993).- La métropole méditerranéenne modèle de la métropole de la modernité 3, Méditerranée, $\mathrm{N}^{\circ} 1.2$, p. $91-94$.

Gizard X., (1993).- Vers l'avenir : la Méditerranće perdue ?, La Méditerranée inquiète, DATAR-AUBE, p. 143-186.

Henry J.R., (1993).- L'Europe du Sud et le Maghreb : le rêve andalou à l'épreuve, in «La Méditerranée, espace de coopération?», Economica, p. 45-57.

Hugon F., (1993).- L'Europe et le Tiers Monde, Revue Tiers Monde, n 136, p. 725-748.

JuAN J.C., (1987).- PACA pôle de croissance européen?, CRCI, Marseille, 36 p.

JunlaRd E., (1976).- Pour une logique des divisions régionales en Europe occidentale, Revue géographique de l'Est, $\mathrm{N}^{\circ} 3.4$, p. 103-120.

NOROIS, (1993).- Arc atlantique, $\mathrm{N}^{\circ} 157,192 \mathrm{p}$.

Ministère de l'Intérieur et de l'Aménagement du Territoire, (1993).- Débat national pour l'aménagement du territoire, Document introductif, DATAR, La documentation française, $124 \mathrm{p}$.

Ruel A., (1991).- L’invention de la Méditerranée, Vingtième Siècle, No 32, p. 7-14.

SCradigli V., (1992).- Européens du Nord, Européens du Sud, Futuribles, mars, p. 35-40.

Voiron-Caniccio C., (1993).- L'arc méditerranéen, espaces, structures et dynamiques, Thèse, Université de Nice, 539 p. 